\title{
El índice ponderal neonatal de fetos sanos en una población de Lima
}

\author{
Ytala Buzzio ${ }^{1}$, Percy Pacora ${ }^{1}$, Wilfredo Ingar ${ }^{1}$, Álvaro Santivañez ${ }^{1}$
}

Resumen

Palabras clave

\begin{abstract}
Objetivo: Establecer los valores del índice ponderal (IP) de un grupo de recién nacidos sanos hijos de mujeres peruanas residentes en Lima, que tuvieron embarazos simples sanos. Materiales y Métodos: Estudio retrospectivo, analítico y transversal, en el cual se revisó la base de datos maternas y perinatales SIP del Departamento de Obstetricia y Pediatría del Hospital Nacional Docente Madre-Niño "San Bartolomé”, entre el 1 de enero de 1991 y el 31 de diciembre de 1999. Se seleccionó los embarazos simples de mujeres sanas entre 20 y 30 años, con IMC entre 20 y 25, sin complicaciones obstétricas, con diferencia \pm 2 semanas entre la fecha de última regla y edad gestacional por examen físico del neonato vivo y sano. Se definió IP como peso al nacer en gramos x 100/[talla en $\mathrm{cm}]^{3}$. Resultados: El índice ponderal [percentil 50 (percentil 10percentil 90)] de 5,443 recién nacidos sanos en Lima para gestaciones de 35,36, 37,38 , 39, 40, 41 y 42 semanas fueron 2,55 (2,22-2,84), 2,59 (2,36-3,04), 2 ,71 (2,423,03), 2,73 (2,44-3,05), 2,72 (2,44-3,05), 2,74 (2,44-3,06), 2,79 (2,45-3,03) y 2,79 $(2,42-3,03) \mathrm{g} / \mathrm{cm}^{3}$, respectivamente. El IP en fetos a término fue mayor en las multíparas que en las nulíparas $(p<0,001)$. No hubo diferencia del IP según sexo. Conclusiones: El feto sano crece en forma simétrica conforme aumenta el tiempo de gestación. El índice ponderal normal varía entre 2,44 y 3,05 g/ $\mathrm{cm}^{3}$. Valores fuera de estos límites nos permiten determinar estados de malnutrición fetal. El índice ponderal del feto sano a término es influenciado por la paridad y el estado nutricional materno y no es influenciado por el sexo.

Peso por estatura; desarrollo fetal; feto.
\end{abstract}

\begin{abstract}
Neonatal ponderal index of healthy fetuses in a population from Lima, Peru

\section{Abstract}

Objetive: To establish values for neonatal ponderal index (PI) in a group of healthy fetuses born from healthy singleton pregnant women at Lima, Peru. Materials and Methods: A retrospective, analytic and transversal study was conducted. Maternal and perinatal data from January 1, 1991 through December 31,1999 was retrieved from the Department of Obstetrics/Gynecology and Pediatrics Database at Hospital Nacional Docente Madre-Niño "San Bartolome". Healthy singleton pregnant women between 20 and 30 years old, BMI between 20 and $25 \mathrm{~kg} / \mathrm{m}^{2}$,
\end{abstract}

1 Departamento de Ginecología Obstetricia y Pediatría. Facultad de Medicina, UNMSM. Lima, Perú.

2 Hospital Nacional Docente Madre-Niño "San Bartolomé". Lima, Perú. without obstetrical complications, with a difference \pm 2 weeks between last menstrual period and gestational age by physical assessment of a live-healthy neonate were selected. PI was defined as birthweight in grams $x$ 100/ [crown-heel length] ${ }^{3}$. Results: The PI [50th percentile (10th percentile- 90th percentile)] in 5,443 Lima healthy neonates at 35, 36, 37, 38, 39, 40, 41, and 42 gestational weeks were 2,55 (2,22-2,84), 2,59 (2,36-3,04), 2,71 (2,423,03), 2,73 (2,44-3,05), 2,72 (2,44-3,05), 2,74 (2,44-3,06), $2,79(2,45-3,03)$, and $2,79(2,42-3,03) \mathrm{g} / \mathrm{cm}^{3}$, respectively. $P I$ of fetuses at term from multiparous women was greater than fetuses from nuliparous women $(p<0,001)$. There was no difference of PI according to gender. Conclusions: Healthy fetuses grow symmetrically during pregnancy. The normal fetal ponderal index is between 2,40 and 3,05 g/ $\mathrm{cm}^{3}$ (10 $0^{\text {th }}$ and $90^{\text {th }}$ percentile). Values out of these limits may diagnose fetal malnutrition. Fetal PI is influenced by parity and maternal nutritional status and not by gender. Keywords: Height weight; fetal development; fetus. 


\section{INTRODUCCIÓN}

A partir de 1960 se ha difundido el empleo de valores de mediciones antropométricas del recién nacido, tales como el peso, la talla, la circunferencia cefálica ${ }^{(1-9)}$.

La determinación del crecimiento fetal normal se basa en la comparación de las medidas antropométricas del neonato con los estándares obtenidos de neonatos "sanos", por provenir de embarazos sin patología detectada. Sin embargo, cuanto más prematuro es un recién nacido, aún sin patología evidente, más sospecha debiéramos tener sobre su crecimiento normal, ya que el parto prematuro es consecuencia de una enfermedad fetal $\left({ }^{10,11}\right)$.

Si bien hay estudios nacionales sobre antropometría neonatal, estos estudios han tenido sesgos en la selección de los pacientes para establecer los criterios de normalidad, tales como la inclusión de gestantes obesas y recién nacidos enfermos $\left({ }^{1-9}\right)$. El índice ponderal constituye una forma de cuantificar el grado de malnutrición (obesidad o desnutrición) del neonato y se representa por la siguiente fórmula: Peso al nacer en gramos x100/(talla en $\mathrm{cm})^{3}$. Esta fórmula se asocia más estrechamente a la mortalidad perinatal que al percentil del peso al nacer con respecto a la edad de gestación $\left({ }^{12-14}\right)$.

La presente investigación tiene como propósito establecer los valores del índice ponderal (IP) de un grupo de recién nacidos sanos hijos de mujeres peruanas residentes en Lima que tuvieron embarazos simples sin complicaciones obstétricas.

\section{MATERIALES Y MÉTODOS}

Se realizó un estudio analítico, retrospectivo, transversal, de la base de datos maternas y perinatales, entre 1 de enero 1991 y el 31 de diciembre de 1999 (SIP), del Departamento de Obstetricia y Pediatría del Hospital Nacional Docente Madre-Niño "San Bartolomé”. A fin de establecer los valores de normalidad, se seleccionó los embarazos simples de mujeres entre 20 y 30 años, con índice de masa corporal entre 20 y $25 \mathrm{~kg} / \mathrm{m}^{2}$, sin patología materna previa y sin complicaciones obstétricas, con variación \pm 2 semanas entre fecha de última regla (FUR) y examen físico del neonato vivo y sano.

Se empleó el programa estadístico SPSS versión 10 (Chicago, Illinois) para establecer los percentiles 10,50 y 90 del índice ponderal del recién nacido sano.

\section{RESULTADOS}

De un total de 47624 nacimientos, se seleccionó $5590(11,7 \%)$ recién nacidos sanos. De estos recién nacidos sólo se obtuvo la información de $5443(97,4 \%)$ para el estudio. La Tabla 1 muestra las características demográficas de las pacientes seleccionadas. Hubo 2674 neonatos femeninos $(49,1 \%)$ y 2769 neonatos masculinos $(50,9 \%)$ El índice ponderal y el sexo del total de recién nacidos son mostrados en la Tablas 2, 3 y 4. No hubo un niño sano menor de 34 semanas de gestación. El IP en los fetos a termino antes de la semana

Tabla 1. Características maternas.

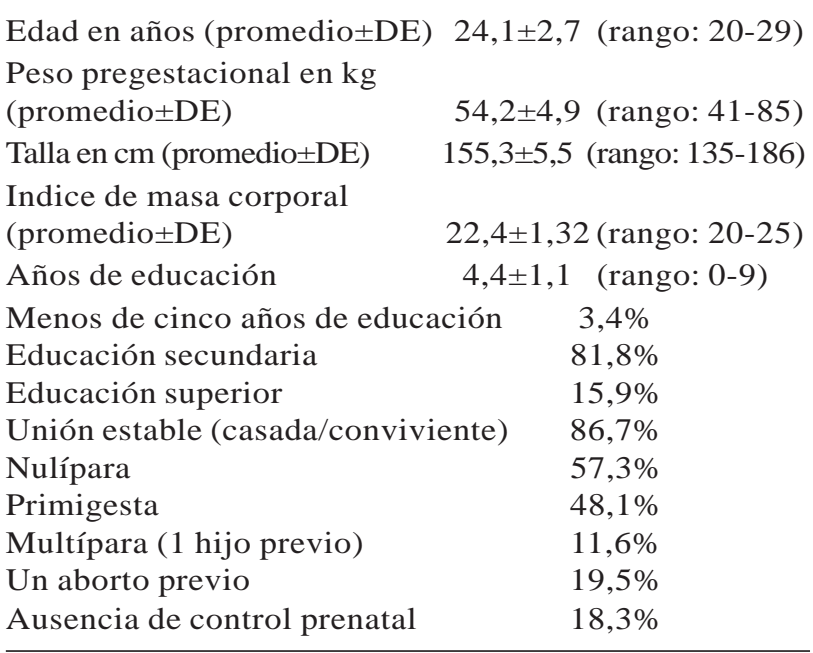


Tabla 2. Índice ponderal neonatal en $\mathrm{g} / \mathrm{cm}^{3}$ de 5443 recién nacidos sanos.

\begin{tabular}{crrrrr}
\hline \multirow{2}{*}{$\begin{array}{c}\text { Edad } \\
\text { gestacional, } \\
\text { semanas }\end{array}$} & Número & \multicolumn{4}{c}{ Percentil } \\
\cline { 3 - 6 } & & 10 & 50 & 90 & 95 \\
\hline 35 & 20 & 2,22 & 2,55 & 2,84 & 2,86 \\
36 & 65 & 2,36 & 2,59 & 3,04 & 3,08 \\
37 & 332 & 2,42 & 2,71 & 3,03 & 3,14 \\
38 & 1123 & 2,44 & 2,73 & 3,05 & 3,16 \\
39 & 1682 & 2,44 & 2,72 & 3,05 & 3,16 \\
40 & 1458 & 2,44 & 2,74 & 3,06 & 3,20 \\
41 & 698 & 2,45 & 2,79 & 3,03 & 3,17 \\
42 & 66 & 2,42 & 2,79 & 3,03 & 3,17 \\
\hline
\end{tabular}

42 fue mayor en las multíparas que en las nulíparas $\left(2,81 \pm 0,5 \mathrm{~g} / \mathrm{cm}^{3}\right.$ versus $2,73 \pm 0,3$ $\left.\mathrm{g} / \mathrm{cm}^{3}, p<0,001\right)$. Tabla 5. Las gestantes multíparas tuvieron mayor índice de masa corporal que las nulíparas $\left(22,5 \pm 1,3 \mathrm{~kg} / \mathrm{m}^{2}\right.$ versus $22,4 \pm 1,3, p<0,01)$. No hubo diferencia según sexo del índice ponderal neonatal en alguna edad gestacional.

\section{DISCUSIÓN}

A fin de establecer los valores de normalidad del crecimiento fetal intrauterino mediante la

Tabla 5. Índice ponderal de 5,443 recién nacidos sanos según paridad.

\begin{tabular}{crllr}
\hline $\begin{array}{c}\text { Edad } \\
\text { gestacional, } \\
\text { semanas }\end{array}$ & \multicolumn{1}{c}{$\begin{array}{c}\text { Número } \\
\text { (n)* }\end{array}$} & \multicolumn{1}{c}{$\begin{array}{c}\text { Multípara } \\
(\mathrm{n})^{*}\end{array}$} & $\begin{array}{c}\text { Valor } \\
\text { de } p\end{array}$ \\
\hline 35 & 20 & $2,51 \pm 0,2(10)$ & $2,56 \pm 0,1(10)$ & 0,6 \\
36 & 65 & $2,66 \pm 0,2(35)$ & $2,62 \pm 0,2(32)$ & $<0,5$ \\
37 & 332 & $2,82 \pm 0,6(164)$ & $2,67 \pm 0,2(171)$ & $<0,01$ \\
38 & 1123 & $2,79 \pm 0,3(501)$ & $2,72 \pm 0,4(633)$ & $<0,01$ \\
39 & 1682 & $2,80 \pm 0,8(675)$ & $2,73 \pm 0,3(1023)$ & $<0,01$ \\
40 & 1458 & $2,82 \pm 0,5(601)$ & $2,73 \pm 0,3(868)$ & $<0,001$ \\
41 & 698 & $2,82 \pm 0,4(267)$ & $2,75 \pm 0,4(435)$ & $<0,05$ \\
42 & 66 & $2,74 \pm 0,2(31)$ & $2,78 \pm 0,2(35)$ & $<0,5$ \\
\hline
\end{tabular}

* En paréntesis numero de casos.
Tabla 3. Índice ponderal de 2674 recién nacidas sanas.

\begin{tabular}{crrcc}
\hline \multirow{2}{*}{$\begin{array}{c}\text { Edad } \\
\text { gestacional, } \\
\text { semanas }\end{array}$} & Número & \multicolumn{3}{c}{ Percentil } \\
\cline { 3 - 5 } & & 10 & 50 & 90 \\
35 & 8 & 2,21 & 2,51 & 2,84 \\
36 & 38 & 2,45 & 2,61 & 3,08 \\
37 & 157 & 2,40 & 2,68 & 3,05 \\
38 & 526 & 2,47 & 2,75 & 3,06 \\
39 & 842 & 2,44 & 2,73 & 3,08 \\
40 & 729 & 2,45 & 2,75 & 3,09 \\
41 & 346 & 2,45 & 2,73 & 3,07 \\
42 & 28 & 2,38 & 2,80 & 3,16 \\
\hline
\end{tabular}

determinación del índice ponderal neonatal (IP), hemos tenido en cuenta las siguientes consideraciones:

1) Hemos seleccionado los embarazos cuya gestación por fecha de menstruación y por examen físico del recién nacido diferían como máximo 2 semanas, debido a que, el considerar la fechas menstruales maternas para el cálculo de la gestación puede inducir a error del rango de hasta 4 semanas, por la existencia de ovulaciones a partir del día 21 de la menstruación y la ocurrencia de sangrado vaginal durante la implantación.

Tabla 4. Índice ponderal de 2,769 recién nacidos varones sanos.

\begin{tabular}{crrcc}
\hline \multirow{2}{*}{$\begin{array}{c}\text { Edad } \\
\text { gestacional, } \\
\text { semanas }\end{array}$} & Número & \multicolumn{3}{c}{ Percentil } \\
\cline { 3 - 5 } & & 10 & 50 & 90 \\
\hline 35 & 12 & 2,27 & 2,55 & 2,82 \\
36 & 27 & 2,24 & 2,57 & 2,84 \\
37 & 175 & 2,44 & 2,72 & 3,00 \\
38 & 597 & 2,43 & 2,72 & 3,03 \\
39 & 839 & 2,44 & 2,71 & 3,02 \\
40 & 729 & 2,42 & 2,72 & 3,02 \\
41 & 352 & 2,47 & 2,74 & 3,06 \\
42 & 38 & 2,42 & 2,78 & 3,03 \\
\hline
\end{tabular}


2) Muchos recién nacidos vivos están enfermos $\mathrm{y}$ fallecen de enfermedades congénitas o desarrollan secuelas neurológicas y/o trastornos en el aprendizaje en la infancia $\left({ }^{10}\right)$. Por tal motivo, sólo hemos seleccionado a los fetos que nacieron vivos y estuvieron sanos en el momento del nacimiento.

3) Debido a que la obesidad materna y el peso materno bajo previo al embarazo son factores de riesgo independiente de la macrosomía fetal $\left({ }^{15}\right)$ y la desnutrición fetal $\left({ }^{16}\right)$, hemos seleccionado en este estudio a las gestantes con índice de masa corporal entre 20 y 25 $\mathrm{kg} / \mathrm{m}^{2}$.

Esta población seleccionada de fetos sanos revela que el ambiente materno era particularmente muy favorable, ya que $97,7 \%$ de las madres tuvo más de 5 años de educación formal, $88,4 \%$ no tenía un hijo previo en casa a quien cuidar, $86,7 \%$ vivía en unión estable con la pareja y $81,7 \%$ tuvo cuidado prenatal.

Lo sorprendente fue que no hubo feto sano menor de 35 semanas en esta población de mujeres jóvenes y sanas en Lima. Este hecho apoya el concepto que el nacimiento prematuro es consecuencia de un proceso patológico que afecta la unidad materno-fetal $\left({ }^{11,17}\right)$.

El IP varió entre 2,40 y $3,05 \mathrm{~g} / \mathrm{cm}^{3}$ (Figura 1 ). Encontramos que normalmente el feto es simétrico (IP entre percentil 10 y 90). Los valores menores de 2,40 nos permite diagnosticar desnutrición fetal y valores mayores de 3,05 obesidad fetal $\left({ }^{18}\right)$. Los neonatos aparentemente sanos de gestaciones pretérmino ( $<37$ semanas) presentan menores valores de IP.

El IP promedio de los recién nacidos muy pretérmino "aparentemente sanos" es menor de $2,4 \mathrm{~g} / \mathrm{cm}^{3}$, señalando que estos recién nacidos sufrían de desnutrición crónica $\left(^{5,9}\right)$. Esta información nos señala que los recién nacidos prematuros tienden a ser generalmente desnutridos, apoyando el concepto que la prematuridad es generalmente la consecuencia de una enfermedad vascular, donde existe una menor distribución de nutrientes al feto $\left({ }^{11,19}\right)$. Esto es particularmente cierto en los recién nacidos muy prematuros $\left({ }^{9}\right)$. Dicho concepto es apoyado por los hallazgos histológicos en la placenta y el lecho placentario de los partos pretérmino, en los que se encuentra lesiones vasculares en el trofoblasto $\left({ }^{20-24}\right)$ y falta de invasión de las células trofoblásticas en el miometrio, a fin de realizar la transformación fisiológica de las arterias espirales que permita la transferencia de nutrientes al concebido $\left({ }^{25,26}\right)$.

El nacimiento prematuro obedecería a una enfermedad fetal de origen multifactorial y la mayoría de los recién nacidos muy prematuros no ha desarrollado todo su potencial de crecimiento genético; presentan detención del crecimiento fetal debido a la presencia de enfermedades asociadas. Por lo tanto, se debe asumir que todo prematuro menor de 35 semanas nace enfermo o desarrollará la enfermedad en el periodo neonatal o en la infancia $\left({ }^{11,17}\right)$.

Los valores del IP del feto a término encontrados por nosotros es similar al de otros autores $\left({ }^{5,9,27}\right)$. Otro hecho notable es que el IP fue mayor en las gestantes multíparas comparado con las nulíparas y no hubo diferencia del índice ponderal de acuerdo al sexo, según ha sido reportado por otros autores $\left({ }^{12,27}\right)$ (Figura 2).

El efecto de la paridad materna sobre el crecimiento fetal parece ser un marcador indirecto del medio ambiente emocional/social y nutricional que tiene el concebido durante su desarrollo $\left({ }^{11,18}\right)$. La multiparidad se asocia a mayor índice de masa corporal de la madre previo al embarazo, el cual, asociado a la ganancia ponderal, contribuyen al mayor desarrollo fetal y aumento en las medidas corporales del feto (peso, talla, perímetro cefálico). Aunque no contamos con la variable ganancia ponderal en este estudio, para realizar el análisis correspondiente de la contribución de esta variable, se ha demostrado que las mujeres multíparas presentan mayor índice de masa corporal que las nulíparas, debido a mayor 


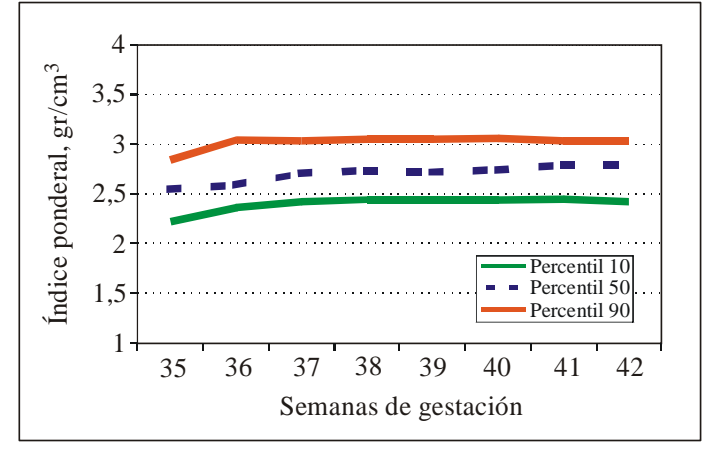

Figura 1. Índice ponderal neonatal de 5,443 fetos nacidos sanos en Lima, 1991-1999.

El feto normalmente es simétrico y mantiene rangos de índice ponderal entre 2,40 y $3,05 \mathrm{~g} / \mathrm{cm}^{3}$.

ganancia ponderal en cada embarazo y aumentan de peso conforme aumentan en edad $\left({ }^{28,29}\right)$.

En otro estudio, nuestro grupo ha demostrado que el IP resulta ser el parámetro más sensible que el peso al nacer de acuerdo a la edad de gestación en la predicción de la mortalidad fetal y neonatal $\left({ }^{14}\right)$. Estos resultados señalan que el feto sano crece simétricamente dependiendo de los nutrientes maternos que recibe e independientemente del sexo fetal que posee. Cuando ocurre el crecimiento anormal de un tejido del organismo, el feto humano se encontraría en mayor riesgo de nacer prematuramente, morir o nacer enfermo $\left({ }^{11}\right)$.

En conclusión, el feto sano crece en forma simétrica conforme aumenta el tiempo de gestación. El índice ponderal fetal normal varía entre 2,44 y $3,05 \mathrm{~g} / \mathrm{cm}^{3}$. Valores fuera de estos límites nos permiten determinar estados de malnutrición fetal. En los fetos sanos, no existe diferencia del índice ponderal de acuerdo al sexo. El índice ponderal del feto sano a término es influenciado por la paridad y el estado nutricional materno.

\section{REFERENCIAS BIBLIOGRÁFICAS}

1. Gruenwald P. Growth of the human fetus. Am J Obst and Gynecol. 1966;94;8:1112-9.
2. Battaglia FC, Lubchenko LO. A practical classification of infants by birthweight and gestational agae. J Pediatr. 1967;71:159-63.

3. Hernández J, Acosta M, Maldonado C, Sacieta L, Meza A. Curva de crecimiento intrauterino. Pediatría UNMSM. 1976;1(1):7-18.

4. Pacora P. Crecimiento fetal en un grupo poblacional de Lima. An Fac Med Lima. 1995;56(2):12-6.

5. Ticona M, Huanco D. Identificación de una nueva población de alto riesgo neonatal con curva de crecimiento intrauterino propias. Rev Peruana Pediatría. 2002;55(3):8-15

6. Velásquez A. Evaluación de las curvas de crecimiento intrauterina usadas en el Perú [Tesis de Especialista en Neonatología]. Lima, Perú: Facultad de Medicina, UNMSM; 2003.

7. Tavares RF. Estudo do crescimento intra-uterino de recém nacidos normais. J Pediatr (Rio J). 1998;74(3):205-12.

8. Fescina RH. Vigilancia antenatal del crecimiento fetal. En: Tecnología Perinatal. Montevideo: Publicación Científica CLAP; 1990. p. 113-31.

9. González R, Gómez R, Castro R, Kae J, Merino P, Etchegaray A y col. Curva nacional de distribución de peso al nacer según edad gestacional. Chile, 1993 a 2000. Rev Méd Chile. 2004;132:1155-65.

10. Naeye R, Dixon J. Distortions in fetal growth standards. Pediat Research. 1978;12:987-91.

11. Pacora P, Romero R. El Parto pretérmino y el recién nacido prematuro. En: Pacheco J (ed). Ginecología y Obstetricia. 2da Edición. Lima: MAD Corp SA; 2005.

12. Walther FJ, Ramaekers LH. The ponderal index as a measure of the nutritional status at birth and its relation to some aspects of neonatal morbidity. J Perinatal Med. 1982;10:42-7.

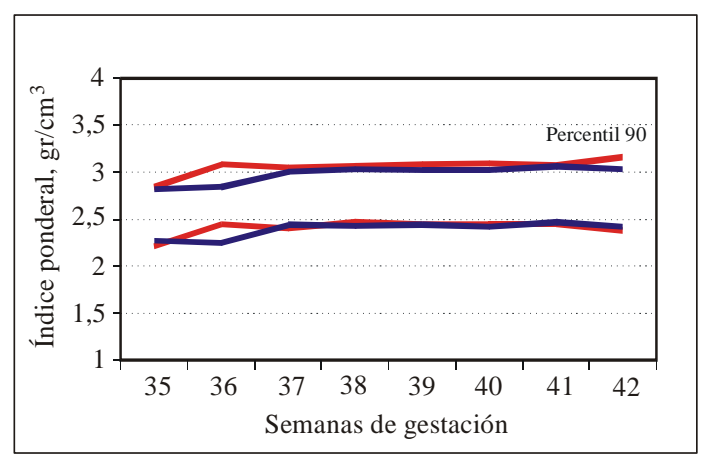

Figura 2. Percentil 10 y 90 del índice ponderal neonatal de 2,764 fetos femeninos sanos y 2,769 fetos masculinos sanos nacidos en Lima, 1991-1999. No existe diferencia significativa en el índice ponderal neonatal entre los fetos masculinos sanos (azul) y los fetos femeninos sanos (rojo) entre el percentil 10 y 90 . 
13. Nimrod CA, Gruslin A. Biology of normal and deviant fetal growth. En: Reece E, Hobbins J. Medicine of the Fetus and Mother. 2nd Edition. Philadelphia: Lippincott-Raven Publishers; 1999. p. 267-78.

14. Pacora P, Ingar W. Fetal ponderal index is more sensitive than birthweight-to-gestational age to predict fetal and neonatal mortality. Am J Obstet Gynecol. 2004;191(6 Suppl 1):S121.

15. Pacora O, Ingar W, Huiza L, Capcha E, Torres R. La obesidad materna es un factor de riesgo materno-perinatal independiente. Rev Asoc Cuerpo Medico Hospital San Bartolomé. 2004;1:24-9.

16. Pacora P. El peso materno anormal previo al embarazo se asocia con mayor riesgo de enfermedad materna y perinatal en Lima. Tema Libre. XV Congreso Peruano de Obstetricia y Ginecología. Lima, Perú; 2005.

17. Romero R, Mazor M, Munoz H, Gomez R, Galasso M, Sherer DM. The preterm labor syndrome. Ann N Y Acad Sci. 1994;734:414-29.

18. Pacora P. Obesidad Fetal. En: Pacheco J (ed). Ginecología y Obstetricia. 2da Edición. Lima: MAD Corp SA; 2005.

19. Huiza L, Pacora P, Santiváñez A, Castro G, Ayala M. La enfermedad perinatal y la prematuridad pertenecen a un síndrome clínico multifactorial: Participación de la herencia de enfermedad vascular, la flora microbiana vaginal y el estado nutricional. An Fac Med Lima. 2003;64;3:167-79.

20. Salafia CM, Ghidini A, Lopez-Zeno JA, Pezzullo JC. Uteroplacental pathology and maternal arterial mean blood pressure in spontaneous prematurity. J Obstet Gynaecol Res. 1998;24(2):141-4.

21. Ghidini A, Salafia CM, Pezzullo JC. Placental vascular lesions and likelihood of diagnosis of preeclampsia. Obstet Gynecol. 1997;90(4 Pt 1):542-5.

22. Salafia CM, Pezzullo JC, Lopez-Zeno JA, Simmens S, Minior VK, Vintzileos AM. Placental pathologic features of preterm preeclampsia. Am J Obstet Gynecol. 1995;173(4):1097-105.

23. Salafia CM, Lopez-Zeno JA, Sherer DM, Whittington SS, Minior VK, Vintzileos AM. Histologic evidence of old intrauterine bleeding is more frequent in prematurity. Am J Obstet Gynecol. 1995;173(4):1065-70.

24. Salafia CM, Minior VK, Pezzullo JC, Popek EJ, Rosenkrantz TS, Vintzileos AM. Intrauterine growth restriction in infants of less than thirty-two weeks' gestation: associated placental pathologic features. Am J Obstet Gynecol. 1995;173(4):1049-57.

25. Kim YM, Bujold E, Chaiworapongsa T, Gomez R, Yoon $\mathrm{BH}$, Thaler HT, Rotmensch S, Romero R. Failure of physiologic transformation of the spiral arteries in patients with preterm labor and intact membranes. Am J Obstet Gynecol. 2003;189(4):1063-9.

26. Kim YM, Chaiworapongsa T, Gomez R, Bujold E, Yoon BH, Rotmensch S, Thaler HT, Romero R. Failure of physiologic transformation of the spiral arteries in the placental bed in preterm premature rupture of membranes. Am J Obstet Gynecol. 2002;187(5):1137-42.

27. Roje D, Ivo B, Ivica T, Mirjana V, Vesna C, Aljosa B, Marko V, Zoran M, Marko M, Tomislav M. Gestational age-the most important factor of neonatal ponderal index. Yonsei Med J. 2004;45(2):273-80.

28. Recktenwald A, Gardiner K, Grim A, Blaskiewicz R, Leet T. Risk of fetal macrosomia among women with weight changes before second birth. Obstetrics and Gynecology. 2003;101(4 Suppl 1):S82.

29. Guihard-Costa AM, Papiernik E, Kolb S. Maternal predictors of subcutaneous fat in the term newborn. Acta Paediatr. 2004;93(3):346-9.

Manuscrito recibido el 02 de marzo de 2005 y aceptado para publicación el 13 julio de 2005.

Correspondencia: Dr. Percy Pacora Portella

Departamento de Gineco-Obstetricia - UNMSM

Hospital Nacional Docente Madre-Niño "San Bartolomé"

Av. Alfonso Ugarte 825. Lima 1, Perú

Correo-e:percypacora@terra.com.pe 